\title{
The Predictive Values of Respiratory Rate Oxygenation Index and Chest Computed Tomography Severity Score for High-Flow Nasal Oxygen Failure in Critically Ill Patients with Coronavirus Disease-2019
}

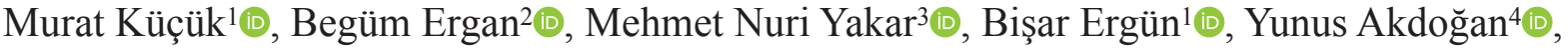

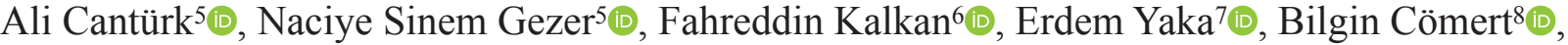 \\ Necati Ali Gökmen
}

\author{
${ }^{1}$ Department of Internal Medicine, Division of Intensive Care, Faculty of Medicine, Dokuz Eylül University, İzmir, Turkey \\ ${ }^{2}$ Department of Pulmonary and Critical Care, Faculty of Medicine, Dokuz Eylül University, İzmir, Turkey \\ ${ }^{3}$ Department of Anaesthesiology and Reanimation, Division of Intensive Care and Faculty of Medicine, Dokuz Eylül University, İzmir, Turkey \\ ${ }^{4}$ Department of Statistics, Faculty of Science, Selçuk University, Konya, Turkey \\ ${ }^{5}$ Department of Radiology, Faculty of Medicine, Dokuz Eylül University, İzmir, Turkey \\ ${ }^{6}$ Department of Actuarial Sciences, Faculty of Science, Selçuk University, Konya, Turkey \\ ${ }^{7}$ Department of Neurology, Faculty of Medicine, Dokuz Eylül University, İzmir, Turkey \\ ${ }^{8}$ Department of Internal Medicine, Faculty of Medicine, Dokuz Eylül University, İzmir, Turkey
}

Background: The prediction of high-flow nasal oxygen (HFNO) failure in patients with coronavirus disease-2019 (COVID-19) having acute respiratory failure (ARF) may prevent delayed intubation and decrease mortality.

Aims: To define the related risk factors to HFNO failure and hospital mortality

Study Design: Retrospective cohort study.

Methods: To this study, 85 critically ill patients ( $\geq 18$ years) with COVID-19 related acute kidney injury who were treated with HFNO were enrolled. Treatment success was defined as the de-escalation of the oxygenation support to the conventional oxygen therapies. HFNO therapy failure was determined as the need for invasive mechanical ventilation or death. The patients were divided into HFNO-failure (HFNO-F) and HFNO-success (HFNO-S) groups. Electronic medical records and laboratory data were screened for all patients. Respiratory rate oxygenation (ROX) index on the first hour and chest computed tomography (CT) severity score were calculated. Factors related to HFNO therapy failure and mortality were defined.

Results: This study assessed 85 patients (median age 67 years, $69.4 \%$ male) who were divided into two groups as HFNO success $(\mathrm{n}=33)$ and HFNO failure $(\mathrm{n}=52)$. The respiratory rate oxygenation (ROX) was measured at 1 hour and the computed tomography (CT) score indicated HFNO failure and intubation, with an area under the receiver operating characteristic of 0.695 for the ROX index and 0.628 for the CT score. A ROX index of $<3.81$ and a CT score of $>15$ in the first hour of therapy were the predictors of HFNO failure and intubation. Age, Acute Physiology and Chronic Health Evaluation II score, arterial blood gas findings "(i.e., partial pressure of oxygen $\left[\mathrm{PaO}_{2}\right]$, $\mathrm{PaO}_{2}$ [fraction of inspired oxygen] $/ \mathrm{SO}_{2}$ [oxygen saturation] ratio)", and D-dimer levels were also associated with HFNO failure; however, based on logistic regression analysis, a calculated ROX on the first hour of therapy of $<3.81$ (odds ratio $[\mathrm{OR}]=4.78,95 \%$ confidence interval $[\mathrm{CI}]=1.75-13.02, P=0.001)$ and a chest $\mathrm{CT}$ score of $>15$ (OR $=2.83,95 \% \mathrm{CI}=1.01-7.88, P=<0.001)$ were the only independent risk factors. In logistic regression analysis, a ROX calculated on the first hour of therapy of $<3.81(\mathrm{OR}=4.78,[95 \% \mathrm{CI}=1.75-13.02], P=$ $0.001)$ and a chest CT score of $>15$ (OR $2.83,95 \% \mathrm{CI}=1.01-7.88, P$ $=<0.001)$ were the independent risk factors for the HFNO failure. The

\footnotetext{
Corresponding author: Begüm Ergan, Department of Pulmonary and Critical Care, Faculty of Medicine, Dokuz Eylül University, İzmir, Turkey e-mail: begum.ergan@deu.edu.tr

Received: July 06, 2021 Accepted: December 12, 2021 Available Online Date: March, 14, 2022 • DOI: 10.4274/balkanmedj.galenos.2021.2021-7-32

Available at www.balkanmedicaljournal.org

ORCID iDs of the authors: M.C. 0000-0003-1705-645X; B.E. 0000-0003-2920-9214; M.N.Y. 0000-0002-3542-3906; B.E. 0000-0003-4828-7576; Y.A. 0000-0003-3520-7493; A.C. 0000-0001-6145-7029; N.S.G. 0000-0002-0868-4545; F.K. 0000-0002-1175-5359; E.Y. 0000-0002-6644-4240; B.C. 0000-0002-2148-5356; N.A.G. 0000-0002-3225-7666

Cite this article as:

Küçük M, Ergan B, Yakar MN, Ergün B, Akdoğan Y, Cantürk A, Gezer NS, Kalkan F, Yaka E, Cömert B, Gökmen NA. The Predictive Values of Respiratory Rate Oxygenation Index and Chest Computed Tomography Severity Score for High-Flow Nasal Oxygen Failure in Patients who are Critically III with Coronavirus Disease-2019. Balkan Med J.; 2022; 39(2):140-7.

Copyright@Author(s) - Available online at http://balkanmedicaljournal.org/
} 
intensive care unit and hospital mortality rates were $80.2 \%$ and $82.7 \%$, respectively, in the HFNO failure group.

Conclusion: The early prediction of HFNO therapy failure is essential considering the high mortality rate in patients with HFNO therapy

\section{INTRODUCTION}

In December 2019, the researchers defined a new coronavirus type called severe acute respiratory syndrome-coronavirus-2 (SARSCoV-2) that causes atypical pneumonia in Wuhan, China. ${ }^{1}$ To date, SARS-CoV-2 rapidly spread, and the disease, coronavirus disease 2019 (COVID-19), caused a pandemic. Viral infectiousness and rapid disease progression to acute respiratory distress syndrome (ARDS) increased the stress on healthcare systems worldwide. This condition contributed to high mortality rates in the early period of the pandemic. ${ }^{2}$ Concerns about aerosolization and the standard lung-protective therapies in ARDS pointed to the early intubation for invasive mechanical ventilation (IMV) in the early period of the pandemic. However, high-flow nasal oxygen (HFNO) became a more popular approach in mild to moderate cases of acute respiratory failure (ARF), owing to the effects of HFNO, including reducing the need for IMV, decreasing complication rates, including ventilator-associated pneumonia, and providing the efficient use of healthcare sources. ${ }^{3}$ Intensive care unit (ICU) professionals may provide heated and moistened oxygen to patients with a $60 \mathrm{~L} / \mathrm{min}$ flow rate and high rates of a fraction of inspired oxygen $\left(\mathrm{FiO}_{2}\right)$ as much as $100 \%{ }^{4}$

The optimal timing of IMV in critically ill patients with COVID-19 is still controversial. Delayed intubation is often related to a bad ARF prognosis and early intubation prevents the lung damage that may occur due to over-ventilation via spontaneous breathing. 5,6 However, a high-flow nasal oxygen (HFNO) and non-IMV (NIMV) therapy prevent complications associated with intubation. ${ }^{7,8}$ Roca et al. ${ }^{9}$ used the respiratory rate oxygenation (ROX) index (arterial oxygen saturation $\left[\mathrm{SaO}_{2}\right] / \mathrm{FiO}_{2} /$ respiratory rate) to show the HFNO effectiveness in patients with pneumonia or ARDS before the COVID-19 pandemic. The researcher highlighted that a calculated ROX value on the second, sixth, and twelfth hour therapy of 4.88 or higher was predictive for HFNO effectiveness. ${ }^{9}$ The studies about the use of HFNO in patients who are critically ill with COVID-19 focused on detecting patients who need IMV using the ROX index. ${ }^{10,11} \mathrm{Hu}$ et al. ${ }^{12}$ determined that the ROX rate of $>5.55$ on the sixth hour of the therapy on day 1 was associated with HFNO success. However, another study revealed that ROX rate of $<5.0$ was related to HFNO failure. ${ }^{13}$ A model including non-respiratory parameters of the Sequential Organ Failure Assessment (SOFA) score and ROX index also had the foresight to detect HFNO failure. ${ }^{14}$ Many kinds of differences in these studies did not achieve a standard modality for an acceptable cut-off value to determine HFNO failure using the ROX index. failure. Using the ROX index and the chest CT severity score combined with the other clinical parameters may reduce mortality. Additionally, multi-centre observational studies are needed to define the predictive value of ROX and chest CT score not only for COVID-19 but also other causes of ARF
The inflammatory markers and chest computed tomography (CT) severity score is associated with short-term outcomes of patients with COVID-19 and correlated with hospital stay and oxygen requirement. ${ }^{15,16}$ Hence, ROX index and chest CT severity score (Each of the five lung lobes was scored on a scale of 0-5 for pulmonary involvement) may be effective to detect HFNO effectiveness during ICU follow-up.

Herein, presented the HFNO experiences in patients with COVID-19. The primary outcome was to determine the factors associated with HFNO failure, and the secondary outcomes were to define the prognostic yield of chest CT severity score and ROX index calculated on the first hour of therapy on the HFNO failure and mortality.

\section{MATERIALS AND METHODS}

\section{Study Population}

This study included all patients with a critical illness $(\geq 18$ years) who are treated only with HFNC for COVID 19-related ARF between July 2020 and November 2021. All patients were treated in a tertiary ICU dedicated to pandemics according to recommendations in the national guideline. COVID-19 diagnosis was confirmed by a real-time reverse transcriptase-polymerase chain reaction test in all patients. Patients younger than 18 years old, treated with NIMV, and alternately treated with HFNO and NIMV were excluded. The study was approved by the local ethics committee of Dokuz Eylül University (2021/03-19) and the Ministry of Health, Republic of Turkey.

\section{Data Collection}

Electronic medical records and laboratory data were screened for each patient and data were collected as follows: age, gender, body mass index, Charlson comorbidity index (CCI), Acute Physiology and Chronic Health Evaluation (APACHE) II, and SOFA scores on ICU admission, and chest CT severity score, arterial blood gas analysis (arterial partial oxygen pressure $\left[\mathrm{PaO}_{2}\right]$; arterial partial carbon dioxide pressure $\left[\mathrm{PaCO}_{2}\right]$; $\mathrm{FiO}_{2} ; \mathrm{PaO}_{2} / \mathrm{FiO}_{2}$ ratio; bicarbonate; $\mathrm{SO}_{2}$; oxygen saturation), laboratory data including hemogram parameters, C-reactive protein (CRP), procalcitonin, lactate dehydrogenase (LDH), alanine aminotransferase (ALT), aspartate aminotransferase (AST), D-dimer, serum creatinine ( $\mathrm{sCr}$ ), total bilirubin, ferritin, high sensitive (HS) troponin I, hospital stay duration, ICU stay, and ICU and hospital mortality. All supportive therapies including renal replacement therapy (RRT), vasopressor use, 
antiviral therapies, immunomodulatory therapies, such as tocilizumab, pulse corticosteroid therapy, thromboembolic prophylaxis including low molecular weight heparin (LMWH), and convalescent plasma therapy, were also recorded.

\section{Definitions}

Severe ARF defined in patients under oxygen therapy with $15 \mathrm{~L} /$ min via non-breathing reservoir bag-mask with an $\mathrm{SO}_{2}$ of $<92 \%$, a respiratory rate of $>30 / \mathrm{min}$, and $\mathrm{a}_{2} \mathrm{O}_{2} / \mathrm{FiO}_{2}$ ratio $<150$. The decision to start HFNO, intubation timing, and IMV was at the initiative of the attending physician following the local guideline. Generally, despite a reasonable $\mathrm{FiO}_{2}$, an $\mathrm{SO}_{2}$ level of $<88 \%$, increased respiratory rate of $>35 / \mathrm{min}$, signs of increased work of breathing, such as the use of accessory respiratory muscles and/ or abdominal paradox, severe metabolic acidosis, and mental changes that compromise upper airway, were the main criteria for the intubation.

The treatment success was defined as de-escalation of HFNO to conventional low-flow oxygen therapies. The HFNO failure was defined as the need for IMV or death. Patients were divided into two groups according to the treatment process as the HFNO failure (HFNO-F) and the HFNO success (HFNO-S) group.

\section{Study Design}

All personal protective equipment, including N95 masks, face shields, goggles, gowns, and gloves, were provided to the ICU staff. Patients wore a surgical mask during HFNO. HFNO was ensured by Airvo ${ }^{\circledR} 2$ (Fisher \& Paykel Healthcare, Irvine, California, USA) or Dräger Evita Infinity V500 (Dräger Medical $\mathrm{GmbH}$; Lübeck, Germany). The therapy was given with a 50-60 $\mathrm{L} / \mathrm{min}$ flow rate, heated air of $31^{\circ} \mathrm{C}-37^{\circ} \mathrm{C}$, and high enough $\mathrm{FiO}_{2}$ to achieve an $\mathrm{SO}_{2}$ value of $>92 \%$. According to local guidelines, all patients were encouraged to return to prone positions during therapy. The therapy gradually reduced in patients in the HFNO-S group and was converted to a simple oxygen mask according to clinical data. The ROX index was calculated only on the first hour of therapy due to the study design.

\section{Chest CT Image Acquisition and Interpretation}

All patients were evaluated before ICU admission with a 64-channel multidetector CT scanner (Brilliance, Philips Medical Systems) with an imaging protocol as follows: 120 $\mathrm{kVp}, 80 \mathrm{~mA}$, slice thickness of $1 \mathrm{~mm}$, and high-spatialfrequency reconstruction algorithm (bone algorithm), without intravenous contrast medium. A scoring system was used to estimate the pulmonary involvement of the CT scans. ${ }^{17}$ Each of the five lung lobes was scored on a scale of 0 to 5 , wherein 0 indicated no involvement, 1 indicated $<5 \%, 2$ indicated $5 \%-25 \%, 3$ indicated $26 \%-49 \%, 4$ indicated $50 \%-75 \%$, and 5 indicated $>75 \%$ involvement. The total CT score was the sum of the individual lobar scores and ranged from 0 to 25 . Atypical CT scans were not scored. Image analysis was performed by a board-certificated radiologist with 15 years of experience in thoracic radiology.

\section{Statistical Analysis}

All continuous variables were expressed as mean \pm standard deviation or median (Interquartile range). Categorical variables were expressed as numbers or percentages. Descriptive statistics for all variables were performed using the Student's t-test, MannWhitney U test, $\chi 2$ test, or Fisher's Exact test.

Univariate and multivariate analyzes were performed to evaluate the variables associated with intubation. The Kaplan-Meier method was used to obtain survival curves, and the different survival probabilities were compared using the log-rank test. The optimal cut-off point for the ROX index and CT score was explored to predict failure of high-flow oxygen therapy by analyzing diagnostic performance with receiver operating characteristic (ROC) curve analysis. A p-value of $<0.05$ was considered significant.

The multivariate logistic regression analysis was used to define independent risk factors for HFNO failure. To build the model, a purposeful selection method was used to select a subset of covariates that were considered clinically important and adjust for confounders and statistical significance. Additionally, the Omnibus test was used to check the goodness of fit, which revealed a $p$-value of 0.001 for the model. The Statistical Package for the Social Sciences Version 24.0 (IBM Corporation, Armonk, NY, USA) was used to perform statistical analysis. The power analysis was determined as at least $80 \%$, with an effect size of 0.8 and alpha of 0.05 for both HFNO-S and HFNO-F groups, to apply the t-test for two independent groups. Power analysis is performed with the Gpower 3.1.9.7 package program $(1-\mathrm{B}=0.95$, alpha $=0.05)$ for 0.8 effect size. With a power of 0.9524 , the sample size to be reached was 35 for each group.

\section{RESULTS}

\section{Patients Characteristics}

We evaluated 316 patients who are critically ill with COVID-19 and are admitted to the ICU. Of them, 85 patients were supported only by HFNO on ICU admission. HFNO was discontinued in 33 $(38.3 \%)$ patients and $52(61.2 \%)$ were intubated due to HFNO failure. The median age was higher in the HFNO-F group than the HFNO-S group (69 [62-79] vs. 62 [53-71] years respectively, $P=$ $<0.001)$. The most common comorbidities include hypertension $(75.3 \%)$, diabetes mellitus $(35.3 \%)$, and coronary artery disease $(23.5 \%)$ in the whole cohort. The APACHE II score (16 [11-24] vs. 11 [9-22], respectively, $P=0.031$ ) and the median chest CT severity score was significantly high in the HFNO-F group than that of the HFNO-S group (16 [13-21] vs. 14 [9-19], respectively, $P=<0.001)$. The ROX rate on the first hour of the therapy was significantly lower in the HFNO-F group than that of the HFNO-S group (3.50 [3.29-3.95] vs. 4.46 [3.49-5.75], respectively, $P=<$ 0.001 ) (Table 1).

\section{Laboratory Data}

No significant difference was found between the groups for hemogram values, procalcitonin, ferritin, $\mathrm{HS}$ troponin I, LDH, sCr, 
and liver function tests (ALT, AST, and total bilirubin). D-dimer was higher in the HFNO-F group than the HFNO-S group (1.45 [1.1-3.2] vs. 0.7 [0.5-1.3] ug/ml, respectively, $P<0.001)$.

The arterial blood gas analysis on the day of ICU admission revealed a similar median $\mathrm{FiO}_{2}$ value (HFNO-F group 0.60 [0.50$60]$ vs. HFNO-S group 0.60 [0.50-60], $P=0.125)$. However, the median $\mathrm{PaO}_{2}$ was lower in the HFNO-F group than the HFNO-S group (61 [48-68] vs. 67 [61-81] mmHg, respectively, $P<0.001)$. The median values of $\mathrm{PO}_{2} / \mathrm{FiO}_{2}(101$ [86-117] vs. 130 [111-152], $P<0.001)$ and $\mathrm{SaO}_{2}(91 \%$ [82-93] vs. 93\% [91-96], $P<0.001)$ were significantly low in the HFNO-F group than that of the HFNO-S group (Table 2).

\section{Treatments and Outcomes}

All therapies including antivirals, tocilizumab, pulse corticosteroid therapy, LMWH, and convalescent plasma did not indicate any differences between the groups. During the ICU stay, the vasopressor use ([80.8\%] vs. [3.0\%], $P<0.001)$ and RRT ([30.8\%] vs. [3.0\%], $P<0,001)$ in the HFNO-F group were higher than the HFNO-S group, but in the HFNO-F group, $92 \%$ and $88 \%$ of patients needed the vasopressors and RRT, respectively, after intubation.

The median duration of HFNO was significantly higher in the HFNO-S group than the HFNO-F group (5 [3-6] vs. 2 [1-4] days, respectively, $P<0.001)$. The median length of ICU stay was longer in the HFNO-F group than the HFNO-S group (11 [6-16] vs. 6 [5-8] days, respectively, $P<0.001)$, but the median hospital length of stay was similar between the groups (HFNO-F group 15 [10-22] vs. HFNO-S group 16 [12-10] days, $P=0.541)$. No deaths were observed in the HFNO-S group; however, in the HFNO-F group, ICU and hospital mortality were $80.2 \%$ and $82.7 \%$, respectively (Table 3).

\section{Risk Factors for HFNO Failure}

The ROX index measured at the first hour after starting therapy and CT score were shown to have good diagnostic performance in predicting the HFNO failure and need for intubation, with an area under the ROC of 0.695 for ROX index and 0.628 for CT score with $95 \%$ confidence intervals (CI) of $0.578-0.811$ and 0.505 0.752 , respectively. The optimal cut-off point of the ROX value that was measured 1 hour after starting treatment was 3.81 and 15 for the CT score (Figure 1,2).

Confounders included in the logistic regression analysis were as follows: ROX score on the first hour of therapy, chest CT severity score, APACHE II score, SOFA score, CCI, and gender. Of which, ROX score on the first hour of therapy was $<3.81$, (Odds Ratio [OR] 4.78, 95\% CI $=1.75-13.02, P=0.001$ ) and

TABLE 1. Clinical Characteristics and Comorbidities

\begin{tabular}{|c|c|c|c|c|}
\hline Characteristics & $\begin{array}{l}\text { All patients } \\
\quad(\mathrm{n}=85)\end{array}$ & $\begin{array}{l}\text { HFNO-S } \\
(\mathrm{n}=33)\end{array}$ & $\begin{array}{l}\text { HFNO-F } \\
(\mathrm{n}=52)\end{array}$ & $P$-value \\
\hline Age & $67(58-75)$ & $62(53-71)$ & $69(62-79)$ & $<0.001$ \\
\hline \multicolumn{5}{|l|}{ Gender } \\
\hline Male & $59(69.4)$ & $23(69.7)$ & $36(69.2)$ & \multirow[t]{2}{*}{1.000} \\
\hline Female & $26(30.6)$ & $10(30.3)$ & $16(30.8)$ & \\
\hline APACHE II score & $15(10-22)$ & $11(9-22)$ & $16(11-24)$ & 0.031 \\
\hline SOFA score $^{1}$ & $4(4-5)$ & $4(4-5)$ & $5(4-6)$ & 0.074 \\
\hline $\mathrm{CCI}$ & $3(2-4)$ & $2(1-4)$ & $4(3-5)$ & 0.072 \\
\hline CT score & $15(11-20)$ & $11(9-15)$ & $19(13-21)$ & $<\mathbf{0 . 0 0 1}$ \\
\hline $\mathrm{ROX}_{\text {score }}^{2}$ & $3.76(3.33-4.69)$ & $4.46(3.49-5.75)$ & $3.50(3.29-3.95)$ & $<0.001$ \\
\hline \multicolumn{5}{|l|}{ Chronic medical illness } \\
\hline Hypertension & $64(75.3)$ & $22(66.7)$ & $42(80.8)$ & 0.198 \\
\hline Diabetes mellitus & $30(35.3)$ & $11(33.3)$ & $19(36.5)$ & 0.813 \\
\hline Coronary artery disease & $20(23.5)$ & $6(18.2)$ & $14(26.9)$ & 0.435 \\
\hline Chronic kidney disease & $9(10.6)$ & $2(6.1)$ & $7(13.5)$ & 0.474 \\
\hline Congestive heart failure & $4(4.7)$ & $1(3.0)$ & $3(5.8)$ & 1.000 \\
\hline Dementia & $4(4.7)$ & $1(3.0)$ & $3(5.8)$ & 1.000 \\
\hline Obstructive pulmonary disease & $11(12.9)$ & $4(2.1)$ & $7(13.5)$ & 1.000 \\
\hline Malignancy & $6(7.1)$ & $2(6.1)$ & $4(7.7)$ & 1.000 \\
\hline Cerebrovascular disease & $3(3.5)$ & $1(3.0)$ & $3(5.8)$ & 0.272 \\
\hline Hyperlipidemia & $8(9.4)$ & $1(3.0)$ & $7(13.5)$ & 0.147 \\
\hline \multicolumn{5}{|c|}{ 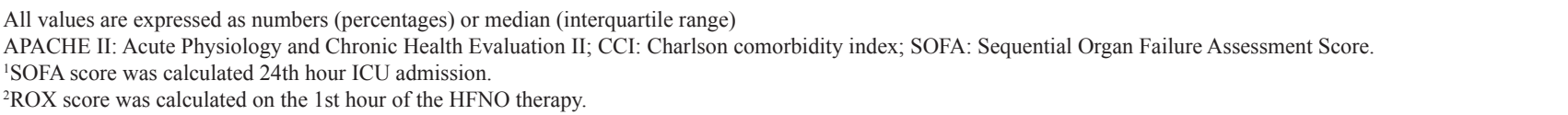 } \\
\hline
\end{tabular}


chest CT score was $>15$ (OR 2.83, 95\% CI $=1.01-7.88, P=$ $<0.001)$ were an independent risk factor for the HFNO failure (Table 4). The sensitivity, specificity, accuracy, and positive and

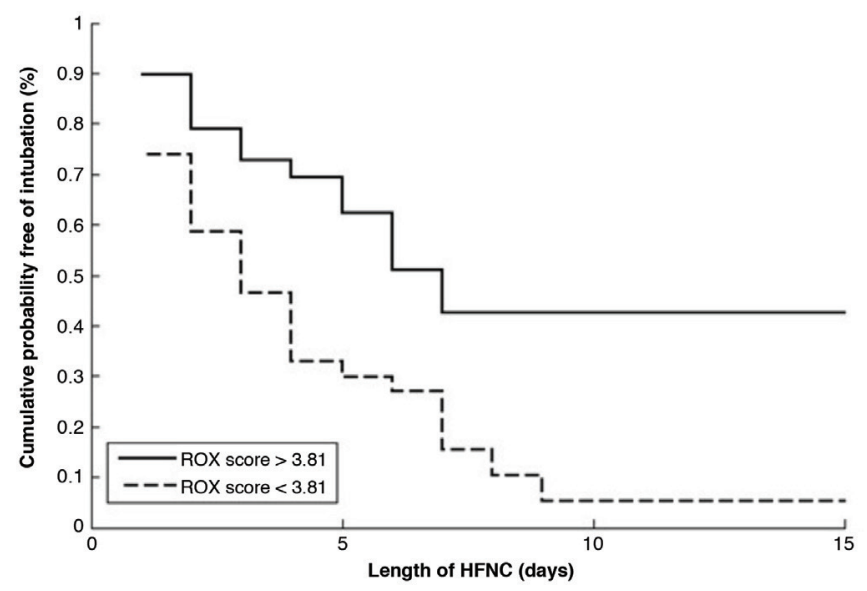

FIG. 1. Kaplan-Meier curve showing the accumulated probability of remaining intubation-free in patients with high-flow nasal cannula according to ROX index measured 1 hour after starting the therapy. negative predictive values of the ROX score are 78.26, 58.97, 69.41, 69.23, and 69.70, respectively, and that of CT score is $65.30,44.44,56.47,61.53$, and 48.48, respectively (Figure 3).

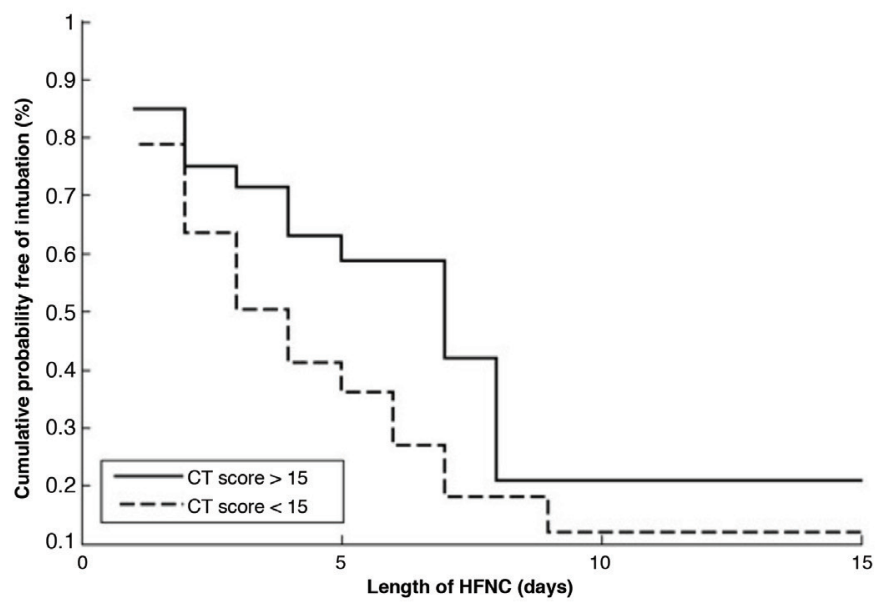

FIG. 2. Kaplan-Meier curve showing the accumulated probability of remaining intubation-free in patients with high-flow nasal cannula according to CT score before ICU admission.

TABLE 2. Laboratory Findings

\begin{tabular}{|c|c|c|c|c|}
\hline Variables & $\begin{array}{l}\text { All patients } \\
\quad(\mathrm{n}=85)\end{array}$ & $\begin{array}{l}\text { HFNO-S } \\
(\mathrm{n}=33)\end{array}$ & $\begin{array}{c}\text { HFNO-F } \\
(\mathrm{n}=52)\end{array}$ & $P$-Value \\
\hline Leukocyte, $10^{3} / \mathrm{Ul}$ & $10.1(7.5-14.1)$ & $10.0(7.2-13.5)$ & $10.1(7.6-15.4)$ & 0.793 \\
\hline Lymphocytes, $10^{3} / \mathrm{UI}$ & $0.5(0.3-0.9)$ & $0.5(0.3-0.7)$ & $0.5(0.4-0.9)$ & 0.484 \\
\hline Hemoglobin, gr/dL & $12.9(11.0-13.9)$ & $12.6(11.8-14.4)$ & $12.7(10.2-13.5)$ & 0.091 \\
\hline $\mathrm{CRP}, \mathrm{mg} / \mathrm{L}$ & $140(78-228)$ & $104(83-186)$ & $157(72-256)$ & 0.073 \\
\hline Procalcitonin, ng/mL & $0.25(0.10-0.49)$ & $0.21(0.08-0.74)$ & $0.34(0.15-0.80)$ & 0.109 \\
\hline $\mathrm{LDH}, \mathrm{U} / \mathrm{L}$ & $543(422-674)$ & $489(385-640)$ & $546(471-732)$ & 0.112 \\
\hline Ferritin, ng/mL & $554(349-975)$ & $638(349-1106)$ & $520(326-820)$ & 0.593 \\
\hline Troponin, ng/mL & $18(8.9-68.1)$ & $11(10.1-19.1)$ & $19.0(10.2-57.2)$ & 0.478 \\
\hline ALT, U/L & $37(24-65)$ & $36(26-85)$ & $38(22-63)$ & 0.407 \\
\hline $\mathrm{AST}, \mathrm{U} / \mathrm{L}$ & $48(35-74)$ & $40(36-73)$ & $51(36-74)$ & 0.391 \\
\hline Total Bilirubin, mg/dL & $0.8(0.6-1.1)$ & $0.7(0.6-1.1)$ & $0.9(0.6-1.3)$ & 0.106 \\
\hline D-dimer, ug/mL & $1.1(0.7-2.4)$ & $0.7(0.5-1.3)$ & $1.45(1.1-.3 .2)$ & $<0.001$ \\
\hline Creatinine $\mathrm{mg} / \mathrm{dL}$ & $1.01(0.77-1.32)$ & $1.00(0.76-1.10)$ & $1.07(0.77-1.32)$ & 0.291 \\
\hline \multicolumn{5}{|c|}{ Arterial Blood Gas Analysis } \\
\hline $\mathrm{pH}$ & $7.45(7.40-7.49)$ & $7.46(7.41-7.49)$ & $7.45(7.37-7.47)$ & 0.062 \\
\hline $\mathrm{PaO}_{2}, \mathrm{mmHg}$ & $62(51-71)$ & $67(61-81)$ & $61(48-68)$ & $<0.001$ \\
\hline $\mathrm{PaCO}_{2}, \mathrm{mmHg}$ & $33(28-37)$ & $32(28-34)$ & $34(28-38)$ & 0.127 \\
\hline $\mathrm{FiO}_{2}, \%$ & $0.60(0.50-60)$ & $0.60(0.50-0.60)$ & $0.60(0.50-0.60)$ & 0.125 \\
\hline $\mathrm{PO} 2 / \mathrm{FiO}_{2}$ & $111(93-132)$ & $130(111-152)$ & $101(86-117)$ & $<0.001$ \\
\hline $\mathrm{SaO}_{2}, \%$ & $92(86-94)$ & $93(91-96)$ & $91(82-93)$ & $<0.001$ \\
\hline $\mathrm{HCO}_{3}, \mathrm{mmol} / \mathrm{L}$ & $24(22-26)$ & $25(21-27)$ & $24(22-25)$ & 0.406 \\
\hline Lactate, $\mathrm{mmol} / \mathrm{L}$ & $1.8(1.4-2.7)$ & $1.8(1.3-2.6)$ & $1.9(1.4-2.7)$ & 0.267 \\
\hline
\end{tabular}

Notes: All values are expressed as numbers (percentages) or median (interquartile range).

ALT: alanine aminotransferase; AST: aspartate aminotransferase; $\mathrm{PaO}_{2}$ : arterial partial oxygen pressure; $\mathrm{PaCO}_{2}$ : arterial partial carbon dioxide pressure; $\mathrm{CRP}$ : C-reactive protein; FiO fraction of inspired oxygen; $\mathrm{HCO} 3$ : bicarbonate; $\mathrm{LDH}$ : lactate dehydrogenase; $\mathrm{SO}_{2}$ : oxygen saturation. 
a

\begin{tabular}{|l|c|c|c|c|c|c|c|}
\hline & AUROC & Sensitivity & Specificity & Accuracy & P.P.V. & N.P.V. & Cut-off points \\
\hline ROX index & 0.695 & 78.26 & 58.97 & 69.41 & 69.23 & 69.70 & 3.81 \\
\hline CT index & 0.628 & 65.30 & 44.44 & 56.47 & 61.53 & 48.48 & 15.00 \\
\hline Abbreviations: P.P.V.: positive predictive value, N.P.V.: negative predictive value \\
\hline
\end{tabular}
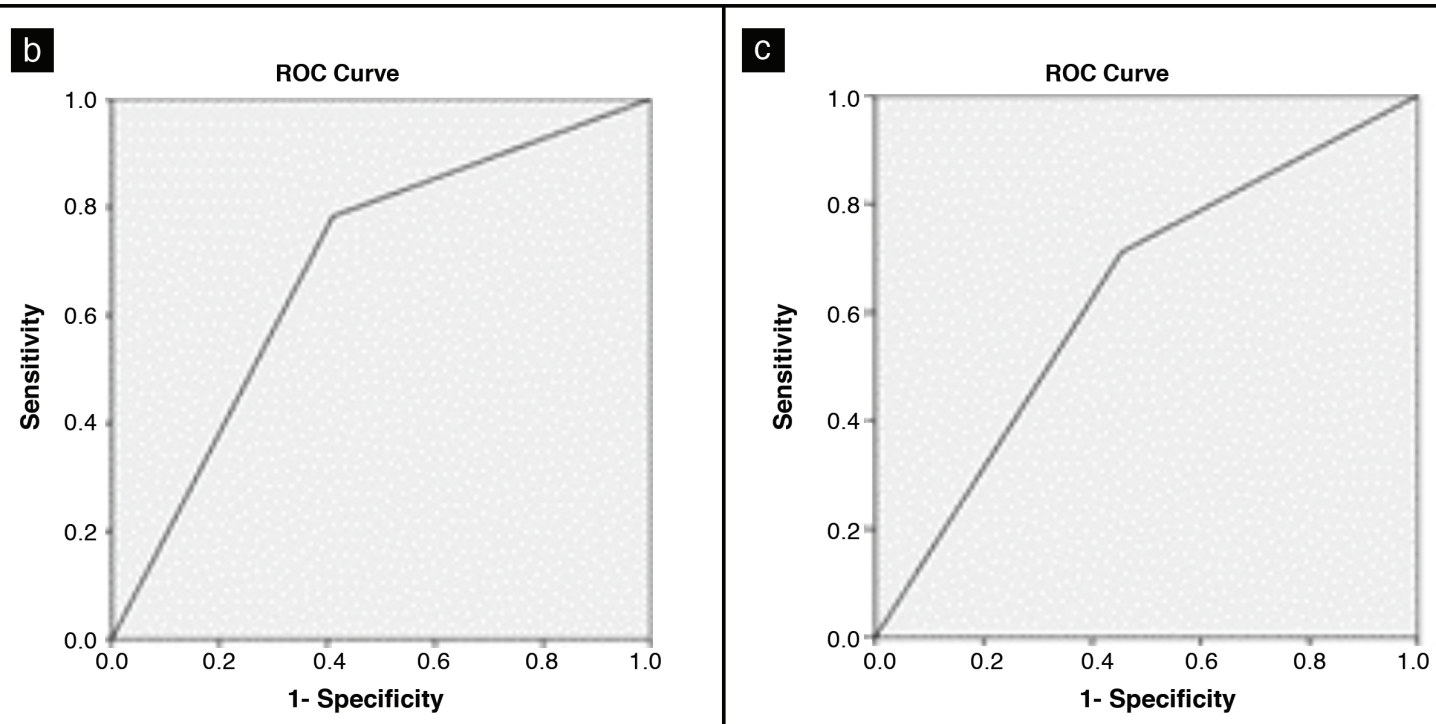

FIG. 3 (a-c). ROC curve analysis results for ROX index and CT score.

TABLE 3. Treatments and Outcomes

\begin{tabular}{|c|c|c|c|c|}
\hline Variables & $\begin{array}{l}\text { All patients } \\
\quad(\mathrm{n}=85)\end{array}$ & $\begin{array}{l}\text { HFNO-S } \\
(\mathrm{n}=33)\end{array}$ & $\begin{array}{l}\text { HFNO-F } \\
(\mathrm{n}=52)\end{array}$ & $P$-Value \\
\hline \multicolumn{5}{|l|}{ Treatments } \\
\hline RRT & $17(20)$ & $1(3.0)$ & $16(30.8)$ & $<0.001$ \\
\hline Vasopressor need during ICU stay ${ }^{1}$ & $43(50.6)$ & $1(3.0)$ & $42(80.8)$ & $<0.001$ \\
\hline Antiviral agents & $85(100)$ & $33(100)$ & $52(100)$ & NA \\
\hline Tocilizumab & $11(12.9)$ & $5(15.2)$ & $6(11.5)$ & 0.744 \\
\hline Pulse corticosteroid ${ }^{2}$ & $40(47.1)$ & $16(48.5)$ & $24(46.2)$ & 1.000 \\
\hline LMWH & $83(97.6)$ & $33(100)$ & $50(96.2)$ & 0.523 \\
\hline Convalescent plasma & $31(36.5)$ & $12(36.4)$ & $19(36.5)$ & 1.000 \\
\hline \multicolumn{5}{|l|}{ Outcomes } \\
\hline HFNO duration, day & $3(2-6)$ & $5(3-6)$ & $2(1-4)$ & $<0.001$ \\
\hline MV duration, day & $1(0-6)$ & 0 & $4(2-12)$ & $\mathbf{N A}$ \\
\hline $\begin{array}{l}\text { Length of duration from the first symptom to ICU } \\
\text { admission, day }\end{array}$ & $8(4-10)$ & $8(5-10)$ & $7(4-10)$ & 0.255 \\
\hline Length of hospital stay before ICU admission, day & $3(1-5)$ & $5(1-6)$ & $2(1-4)$ & 0.246 \\
\hline Length of hospital stay, day & $15(11-21)$ & $16(12-19)$ & $15(10-22)$ & 0.541 \\
\hline Length of ICU stay, day & $8(5-14)$ & $6(5-8)$ & $11(6-16)$ & $<0.001$ \\
\hline Hospital mortality & $43(50.6)$ & 0 & $43(82.7)$ & NA \\
\hline ICU mortality & $42(49.4)$ & 0 & $42(80.2)$ & NA \\
\hline \multicolumn{5}{|l|}{$\begin{array}{l}\text { All values are expressed as numbers (percentages) or median } \\
\text { HFNO: high-flow nasal oxygen; ICU: intensive care unit; IM } \\
\text { therapy. } \\
{ }^{1} \text { Norepinephrine of }>0.15 \mu \mathrm{g} / \mathrm{kg} / \mathrm{min} \\
{ }^{2} \text { Pulse corticosteroid (methylprednisolone) of } \geq 250 \mathrm{mg} / \text { day. }\end{array}$} \\
\hline
\end{tabular}


TABLE 4. Risk Factors Associated With HFNO Failure in the Multivariate Regression Analysis Among Patients With COVID-19.

\begin{tabular}{|c|c|c|}
\hline & OR $(95 \% \mathrm{CI})$ & $P$-value \\
\hline ROX Index of $<3.811$ & $4.78(1.75-13.02)$ & $<0.001$ \\
\hline Chest CT Score of $>15$ & $2.83(1.01-7.88)$ & $<0.001$ \\
\hline Sex & $0.73(0.21-2.54)$ & 0.621 \\
\hline Charlson Comorbidity Index & $1.18(0.83-1.68)$ & 0.352 \\
\hline APACHE II Score & $1.01(0.93-1.10)$ & 0.804 \\
\hline SOFA Score & $1.04(0.59-1.83)$ & 0.883 \\
\hline \multicolumn{3}{|c|}{$\begin{array}{l}\text { APACHE: Acute Physiology and Chronic Health Evaluation; CT: computed } \\
\text { tomography; SOFA: Sequential Organ Failure Assessment; OR: Odds ratio. } \\
{ }^{1} \text { Calculated on the first hour of HFNO. }\end{array}$} \\
\hline
\end{tabular}

\section{DISCUSSION}

The present study evaluated the risk factors for HFNO failure and concluded three main results. First, the treatment failure with HFNO was $61.1 \%$ in severe ARF. Second, HFNO failure was strongly associated with mortality, wherein ICU and hospital mortality were $80.2 \%$ and $82.7 \%$, respectively. Third, ROX index and chest $\mathrm{CT}$ severity score were independent risk factors for HFNO failure in patients who are critically ill with COVID-19 and are initially treated with HFNO. The results revealed that the value of the calculated ROX index on the first hour of therapy was $<3.81$, which indicated a 4.78-fold increased therapy failure. However, a chest CT severity score of $>15$ pointed to a 2.83 -fold increased therapy failure.

During the COVID-19 pandemic, some researchers offered the use of HFNO after risk and benefit analysis not only to avoid intubation but also to provide rational use of limited healthcare sources. ${ }^{3}$ Hence, the strategy, which lets permissive hypoxemia in COVID-19 patients under close monitoring, came to the fore. In the ICU practice, the prediction of HFNO failure is a difficult issue to overcome in patients with ARF. However, our study revealed that $\mathrm{PaO}_{2} / \mathrm{FiO}_{2}$ ratio was lower in patients with HFNO failure. ${ }^{10,18}$ Previous studies revealed the intubation requirement in most patients with ARF $(30 \%-40 \%)$ who are initially treated with HFNO ${ }^{19,20}$ However, a study that included patients, whose chest CT scores were similar, revealed a better ICU survival (56\% vs. $80 \%$ ) and hospital survival (65\% vs. $100 \%)$ in patients intubated in the first 48 hours than those intubated after 48 hours. ${ }^{21}$ Additionally, a previous study that included patients with ARDS revealed that delayed intubation (1 day or longer after admission) was associated with a worse outcome compared with early intubation/no need for intubation. ${ }^{22}$ This present study revealed that the median length of HFNO was 2 days, and the mortality rate was high after intubation in the HFNO failure group, as in previous studies. Therefore, the individualization and continuity of treatment and close monitoring to avoid delayed intubation are the key elements.

Having the tools, which are useful to predict the failure of therapies administered before intubation, is essential to avoid delayed intubation. The parameters, D-dimer and $\mathrm{PaO}_{2} / \mathrm{FiO}_{2}$ ratio, which indicate disease severity, were low in the HFNO-F group, as in a previous study. Additionally, the median age was higher in the HFNO-F group in the study. ${ }^{10}$ Clinical signs and laboratory data in patients with permissive hypoxemia are essential to detect disease progression. Calculated ROX index at different times of therapy was useful in predicting HFNO effectiveness. The literature reported many kinds of studies that determined the predictive value of the ROX index for the HFNO failure, but no consensus was made for the calculation time and cut-off value of the index. A study that included 272 patients with COVID-19 revealed a $39.7 \%$ rate of HFNO failure, and all these patients underwent intubation. ${ }^{23}$ In this group, the mortality rate was $45.4 \% .^{24}$ Additionally, the researcher revealed a value of ROX index of $>3.67$ on the twelfth hour of therapy as an accurate predictor of successful HFNO weaning. ${ }^{23}$ Roca et al. ${ }^{9}$ identified the ROX index calculated in the early period of the therapy as a strong HFNO predictor. A value of ROX index of $>4.88$ was related to decreased intubation probability in their cohort. Hu et al. ${ }^{12}$ revealed that a ROX index of $>5.5$ after the sixth hour of initiation was related to HFNO success. A previous study that included 293 patients with COVID-19 revealed the following ROX indexes: $<2.85$ for the second hour, $<3.37$ for the sixth hour, and $<3.85$ for the twelfth hour of therapy, as a predictor for HFNO failure. The researchers also revealed an overall mortality rate of $53 \%$. The mortality rate was $71 \%$ in patients who underwent intubation. Contrarily, patients who are successfully weaned from HFNO had a 93\% survival. ${ }^{10}$ Similarly, in this present study, a ROX value on the first hour of therapy of $<3.81$ was related to HFNO failure. The HFNO failure rate was $61.1 \%$ in our study, and the mortality was $80.2 \%$ in this group. However, all patients with HFNO success survived.

Chest CT severity score has a high sensitivity for diagnosing patients with COVID-19. Thus, the score has been used for the management of patients since the COVID-19 pandemic started. ${ }^{24} \mathrm{~A}$ study that investigated the association between the semi-quantitative score based on lung involvement in chest CT and clinical stage of disease revealed a chest CT score of 18 or higher with a high predictive value for short-term mortality. ${ }^{16}$ Additionally, chest CT score was correlated with D-dimer and CRP levels. ${ }^{16}$ A cohort that included 236 patients with COVID-19 revealed a positive correlation between the chest CT score and ICU requirement and mortality. ${ }^{25}$ The present study revealed a $>15$ chest CT score, which increased the risk for HFNO failure and was associated with increased mortality. Our study has novel findings, as chest CT score may be used as another additional tool for HFNO failure prediction.

Our study has some limitations. First, the results of this study cannot be generalized since the study provided single-center data. HFNO failure and intubation decisions are not dependent on a clinical protocol, and differences between expert clinicians in terms of interpretation and application could not be predicted. Second, because of the extreme workload due to the pandemic, only the ROX index on the first hour of therapy was evaluated. Additionally, our study population was relatively small to analyze composite indexes, such as combined use of ROX index and CT score, thus cannot be generalizable for patients treated with both. Lastly, the predictive yield of ROX index and CT score cut-off 
values to identify patients with possible HFNC failure is relatively limited. However, the study has some strengths. We have evaluated the efficacy of HFNO in a very severe ARF cohort and, as we know, it is the first study to evaluate ROX index and CT score together in detecting HFNO failure.

In conclusion, the study presented the risk factors for HFNO failure in patients who are critically ill with COVID-19. We have tried to define a simple cut-off value for a practical bedside approach to recognize critical patients as early as possible to prevent delayed intubation, and therefore mortality, in the era of pandemics with high ICU workload. In this setting, early recognition of patients with a possible risk for HFNO failure is crucial. Together with other clinical parameters, we suggest that a ROX index of $<3.81$ or a CT score of $>15$ may be helpful for the clinical decision-making process. The diagnostic yield of combined use of both scores as a composite index and the assessment of optimum threshold values of these indices for HFNO failure merits further research.

Ethics Committee Approval: The study was approved by the local ethics committee of Dokuz Eylül University (2021/03-19) and the Ministry of Health, Republic of Turkey.

Data Sharing Statement: The data that support the findings of this study are available from the corresponding author upon reasonable request.

Author Contributions: Concept- M.K., B.Ergan, M.N.Y., B.Ergün, Y.A., A.C., N.S.G., F.K., E.Y., B.C., A.N.G.; Design- M.K., B.E., M.N.Y., B.E., Y.A., A.C., N.S.G., F.K., E.Y., B.C., N.A.G.; Supervision - B.E., E.Y., B.C., A.N.G.; Data Collection and Processing M.K.; Analysis and/or Interpretation - M.K., B.E., M.N.Y., B.E., Y.A., A.C., N.S.G., F.K.; Writing - M.K., B.E., M.N.Y., B.E., Y.A., A.C., N.S.G., F.K., E.Y., B.C., A.N.G.

Conflict of Interest: No conflict of interest was declared by the authors.

Funding: The authors declared that this study received no financial support.

\section{REFERENCES}

1. Zhu N, Zhang D, Wang W, et al. A Novel Coronavirus from Patients with Pneumonia in China, 2019. N Engl J Med. 2020;382:727-733. [Crossref]

2. Huang C, Wang Y, Li X, et al. Clinical features of patients infected with 2019 novel coronavirus in Wuhan, China. Lancet. 2020;395:497-506. [Crossref]

3. Cinesi Gómez C, Peñuelas Rodríguez Ó, Luján Torné ML, et al. Clinical Consensus Recommendations Regarding Non-Invasive Respiratory Support in the Adult Patient with Acute Respiratory Failure Secondary to SARS-CoV-2 infection. Med Intensiva (Engl Ed). 2020;67:261-270. [Crossref]

4. Rochwerg B, Granton D, Wang DX, et al. High flow nasal cannula compared with conventional oxygen therapy for acute hypoxemic respiratory failure: a systematic review and meta-analysis. Intensive Care Med. 2019;45:563-572. [Crossref]

5. Gattinoni L, Chiumello D, Caironi P, et al. COVID-19 pneumonia: different respiratory treatments for different phenotypes?. Intensive Care Med. 2020;46:10991102. [Crossref]

6. de Simone G, Mancusi C. COVID-19: Timing is Important. Eur J Intern Med. 2020;77:134-135. [Crossref]

7. Tobin MJ, Laghi F, Jubran A. Caution about early intubation and mechanical ventilation in COVID-19. Ann Intensive Care. 2020;10:78. [Crossref]
8. Rola P, Farkas J, Spiegel R, et al. Rethinking the early intubation paradigm of COVID-19: Time to change gears?. Clin Exp Emerg Med. 2020;7:78-80. [Crossref]

9. Roca O, Caralt B, Messika J, et al. An index combining respiratory rate and oxygenation to predict outcome of nasal high-flow therapy. Am J Respir Crit Care Med. 2019;199:1368-1376. [Crossref]

10. Calligaro GL, Lalla U, Audley G, et al. The utility of high-flow nasal oxygen for severe COVID-19 pneumonia in a resource-constrained setting: A multi-centre prospective observational study. EClinicalMedicine. 2020;28:100570. [Crossref]

11. Ferrando C, Mellado-Artigas R, Gea A, et al. Awake prone positioning does not reduce the risk of intubation in COVID-19 treated with high-flow nasal oxygen therapy: A multicenter, adjusted cohort study. Crit Care. 2020;24:597. [Crossref]

12. Hu M, Zhou Q, Zheng R, et al. Application of high-flow nasal cannula in hypoxemic patients with COVID-19: a retrospective cohort study. BMC Pulm Med. 2020;20:324. [Crossref]

13. Patel M, Chowdhury J, Mills N, et al. ROX index predicts intubation in patients with COVID-19 pneumonia and moderate to severe hypoxemic respiratory failure receiving high flow nasal therapy. medRxiv. 2020. medRxiv preprint doi: https://doi. org/10.1101/2020.06.30.20143867. [CrossRef]

14. Mellado-Artigas R, Mujica LE, Ruiz ML, et al. Predictors of failure with highflow nasal oxygen therapy in COVID-19 patients with acute respiratory failure: a multicenter observational study. J Intensive Care. 2021;9:23. [CrossRef]

15. Saeed GA, Gaba W, Shah A, et al. Correlation between Chest CT severity scores and the clinical parameters of adult patients with COVID-19 pneumonia. Radiol Res Pract. 2021;2021:6697677. [CrossRef]

16. Francone M, Iafrate F, Masci GM, et al. Chest CT score in COVID-19 patients: correlation with disease severity and short-term prognosis. Eur Radiol. 2020;30:68086817. [CrossRef]

17. Chang YC, Yu CJ, Chang SC, et al. Pulmonary sequelae in convalescent patients after severe acute respiratory syndrome: Evaluation with thin-section CT. Radiology. 2005;236:1067-1075. [CrossRef]

18. Panadero C, Abad-Fernández A, Rio-Ramirez MT, et al. High-flow nasal cannula for acute respiratory distress syndrome (ARDS) due to COVID-19. Multidiscip Respir Med. 2020;15:693. [CrossRef]

19. Frat JP, Ragot S, Thille AW. High-flow nasal cannula oxygen in respiratory failure. N Engl J Med. 2015;373:1374-1375. [CrossRef]

20. Messika J, Ben Ahmed K, Gaudry S, et al. Use of high-flow nasal cannula oxygen therapy in subjects with ARDS: A 1-year observational study. Respir Care. 2015;60:162-169. [CrossRef]

21. Bauer PR, Gajic O, Nanchal R, et al. Association between timing of intubation and outcome in critically ill patients: a secondary analysis of the ICON audit. J Crit Care. 2017;42:1-5. [CrossRef]

22. Kangelaris KN, Ware LB, Wang CY, et al. Timing of intubation and clinical outcomes in adults with acute respiratory distress syndrome. Crit Care Med. 2016;44:120-129. [CrossRef]

23. Chandel A, Patolia S, Brown AW, et al. High-flow nasal cannula therapy in COVID-19: using the ROX index to predict success. Respir Care. 2021;66:909-919. [CrossRef]

24. Ai T, Yang Z, Hou H, et al. Correlation of chest CT and RT-PCR testing for Coronavirus disease 2019 (COVID-19) in China: a report of 1014 cases. Radiology. 2020;296:E32-E40. [CrossRef]

25. Colombi D, Bodini FC, Petrini M, et al. Well-aerated lung on admitting chest CT to predict adverse outcome in COVID-19 pneumonia. Radiology. 2020;296:E86-E96. [CrossRef] 